\section{Motivational properties of performance feedback ${ }^{1}$}

PAUL G. SWINGLE, MCMaster University, Hamilton, Ont., Canada

Ss, exposed to a bogus performance norm, lever-pressed to accumulate a point score. Although group results indicated that easy and difficult norms enhance performance speed, the effect was shown to be limited primarily to the slowest responders. Fast responders' speeds were maintained by simple performance feedback in the absence of externally imposed norms.

Earlier studies (Swingle, Coady, \& Moors, 1966; Swingle \& Moors, 1967) indicate that in a lever-pressing situation, with only performance feedback and no material incentive, at least two distinct classes of performers are apparent: those who persistently increase their level of performance and those who stabilize at a relatively low level of performance. Fast responders appear to respond to the self-competitive aspects of the situation and are continually attempting to better their previous performance record.

An interesting question is that of the effect of arbitrary performance norms of varying difficulty upon the performance of fast and slow responders in a simple lever-pressing situation. If fast responders are, in fact, optimally motivated by the self-competitive aspect of the performance situation, easy goals or norms should have no effect, since, once attained, the self-competitive process should become prepotent (i.e., the norm may be irrelevant in that it is lower than the fast responder's self-imposed standard). Thus, fast responders, given an easy norm, should perform at about the same speed as those given no norm other than a "do your best" induction.

If, however, one assumes that there is an optimal level of activation for performance tasks (Leuba, 1955), the effect of an impossible or extremely difficult norm could be to actually decrease the level of performance of fast responders. A study by Swingle \& Moors (1967), for example, demonstrated that added incentive may actually have a suppressive effect upon the performance rate of fast responders. Thus, if the norm is too demanding, it may disrupt the orderly progression of the standard toward higher performance levels for fast performers.

Fig. 1. Mean change in lever-pressing speed as a function of seven five-trial blocks for all Ss without speed distinction.
The present study explored the effect of easy and of impossible norms upon the performance of fast and slow responders in a simple lever-pressing situation, with performance feedback.

OVERVIEW OF THE

EXPERIMENTAL SITUATION

The Ss were seated in ventilated $4 \times 6 \mathrm{ft}$ cubicles, acoustically isolated from the programming and recording apparatus. The cubicles contained a standard $19 \times 21$ in. table-model relay rack, upon which one light, two electric impulse counters, and a Stromberg Carlson cam key (No. 171D) were mounted. The experimental session consisted of a series of 15 practice trials (20 sec each) and $35 \quad 20-\mathrm{sec}$ test trials. During the time-in period, Ss' lever-pressing responses were reinforced on a FR5 schedule, with points accurnulated on the counter. All trials were indicated by the onset of the rack-mounted light, and the $20-\mathrm{sec}$ time-in was timed from the S's first lever press. During the trial, the $S$ could accumulate points as shown on the impulse counter labeled "POINTS." The second impulse counter (labeled "TRIALS") recorded the number of trials completed. Between each trial, there was a rest interval that averaged $20 \mathrm{sec}$ in length and varied between 10 and $30 \mathrm{sec}$. Between the practice trials and the test trials there was a pause of approximately $70 \mathrm{sec}$. Stimulus presentations, reinforcement points, recording of lever-pressing responses, etc., were all accomplished by means of standard programming and recording apparatus.

Once seated in front of the response panel, the $S$ heard tape-recorded

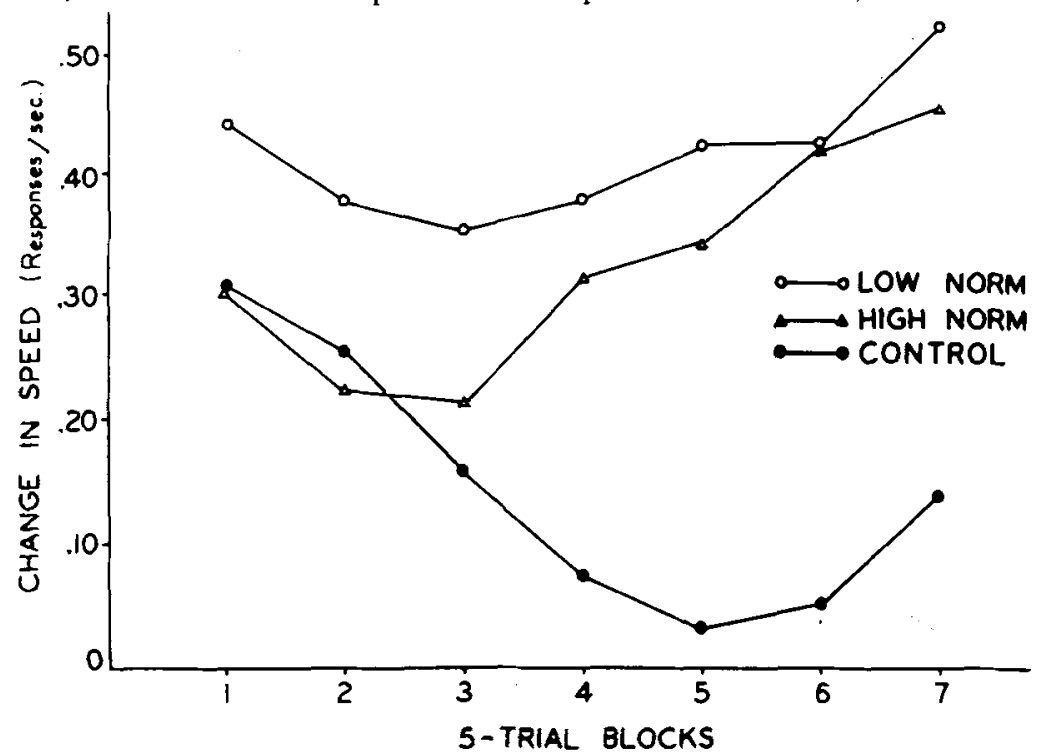

instructions over an intercom system. All Ss were told that the study was concerned with "motor activity" and that they could accumulate points, as shown on the counter, if they pushed the lever rapidly whenever the "GO" light on their panel was illuminated and that the faster they manipulated the lever, the more points they would receive.

\section{SUBJECTS}

Ninety male college students, registered in the introductory psychology course or introductory commerce course at McGill University or Sir George Williams University in Montreal, comprised the sample.

\section{PROCEDURE}

The experimental design consisted of three groups of $30 \mathrm{Ss}$ each. The groups differed with respect to the performance norm communicated to them by the $\mathrm{E}$ at the end of 15 "practice" trials. Within each group of 30 , the Ss were further subdivided into three groups of $10 \mathrm{Ss}$ each, based upon their lever-pressing speed during the last 5 practice trials. Thus, the experiment consisted of nine groups of $10 \mathrm{Ss}$ each as defined by a 3 by 3 factorial design with three levels of initial response speed and three norm conditions: no norm (control), easy norm (low norm), and an impossible norm (high norm).

After Ss had completed 15 preliminary trials, the experimental treatment instructions were presented via the intercom system after a delay of $70 \mathrm{sec}$. All Ss were told that the previous series of trials were "practice trials in order to familiarize you with the task," and that "now the actual test is to begin." In the control conditions, Ss were simply told again to "try to accumulate as many points as possible in the next 35 trials." For both experimental conditions, the low norm and 
Fig. 2. Mean change in lever-pressing speed for the slow responders in each group as a function of seven five-trial blocks.

the high norm, the performance norm was based upon the Ss' own actual response rates during the last 5 -trial block of their practice-trial series. Ss in the low-norm condition received (verbally) a comparison score which they could exceed, if they maintained their average response rate of the last 5-trial practice block, at approximately Trial 25 , or 10 trials before the end of the 35-trial series. Ss in the high-norm condition were given a comparison score based upon their average performance in the last 5-trial practice block which, at that average speed, would require 45 trials to attain, or 10 more than the scheduled 35-trial series. ${ }^{2}$ In both norm conditions, Ss were told: "To give you an idea of how well you are doing, we have found that most males your age obtain, on the average, (X) points in the next 35 trials, or about $(X / 35)$ points per trial. Try to accumulate as many points as possible." In addition to the overall standard score, Ss were also given an approximate trial-by-trial score (to one decimal place) as indicated by the value of $\mathrm{X} / 35$ in the above statement. Thus, $\mathrm{S}$ could, by referring to the trials counter, determine the approximate relationship between his score and the norm at any time during the session.

\section{RESULTS AND DISCUSSION}

The response data were converted to difference scores for each of the seven five-trial blocks during which the experimental manipulation was in effect. The difference scores were obtained by subtracting the S's base response rate (estimated by the five-trial block average for Practice Trials 11 through 15) from each S's response rate over the course of the 35-trial experimental series. The difference data shown as mean change in lever-pressing speed for all $30 \mathrm{Ss}$ in each one of the three conditions are shown in Fig. 1. The curves indicate an increase in speed for all groups at the outset of the test-trial series. This increment probably results from the 70-sec break between practice and test trials and from the instructions specifying that the future trials constituted the actual test.

It is interesting to note that without distinguishing Ss on the basis of pretreatment speed scores, both the high-norm and the low-norm conditions

Fig. 3. Mean change in lever-pressing speed for the intermediate responders in each group as a function of seven five-trial blocks.

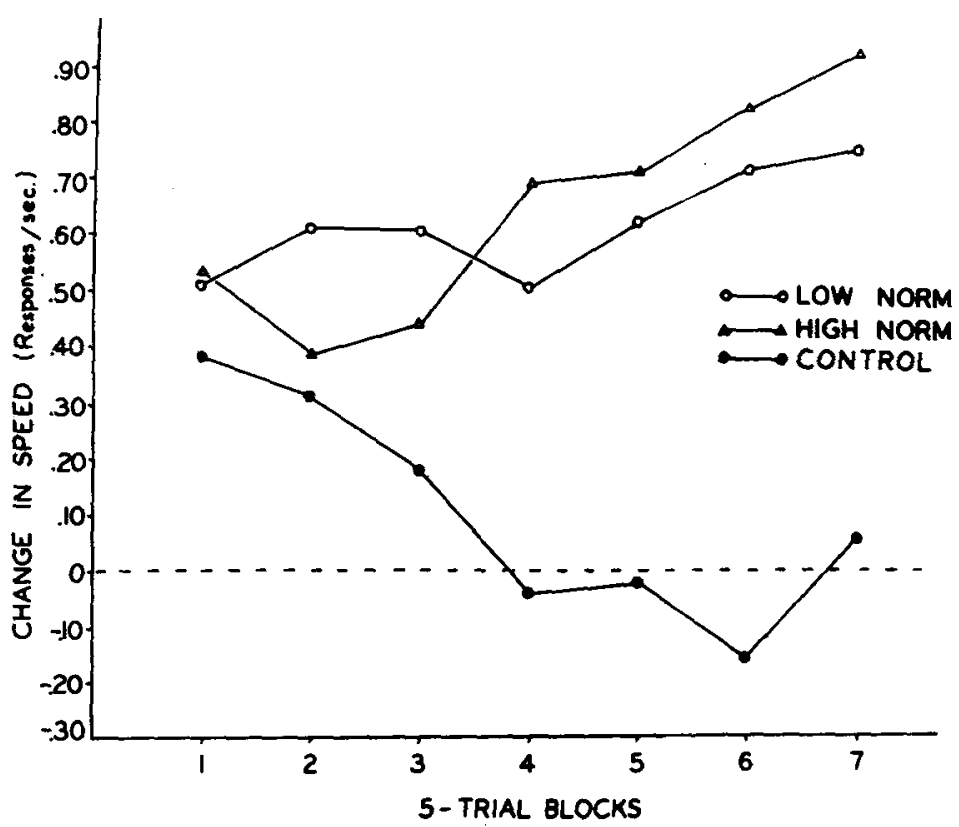

show, after an initial decline, a consistent increase in speed over the course of the 35 trials. The control condition, on the other hand, shows a consistent reduction in speed, which, at Trial Block 5, is practically equal to the base line established during the last 5 practice trials. It is also interesting to note that the low-norm condition is consistently higher than the high-norm condition over the entire 35 trials.

The data shown in Fig. 1 were submitted to analysis of variance. Neither the norm $[F(2,87)=2.72, p<.10]$ nor the trials $[F(6,522)=1.98, p<.10]$ main effects exceed the conventional level of significance, whereas the interaction between trials and norms is reliable $[\mathrm{F}(12,522)=1.86, \mathrm{p}<.05]$.

Simple comparisons indicate that control-group Ss are, on the average, significantly slower than the Ss in both the high-norm and low-norm conditions after Trial Block $3(\mathrm{p}<.05)$.

The groups of $30 \mathrm{Ss}$ were then divided into subgroups of 10 responders each according to the S's baseline speed. Thus, one group of 10 fast responders (mean base rate $=6.5$ responses $/ \mathrm{sec}$ ), one group of 10 slow responders $(\bar{X}=4.8)$, and one group of 10 responders halfway between the two extremes $(\overline{\mathrm{X}}=5.8)$ were obtained. The mean difference-score data for the slow (SLO), intermediate (ITM), and fast (FST) responders over the course of the 35-trial session are shown in Figs. 2, 3, and 4. It is immediately apparent from the data shown in the figures that the effect of norms, as compared to the control condition, reflected in the group total for 30 Ss not distinguished according to initial base speed (Fig. 1), is attributable primarily to the SLO group. The FST group, on the other hand, show very little effect attributable to the different treatment conditions. SLO responders (Fig. 2) show an initial increment in speed over the base rate during the first post-practice trial

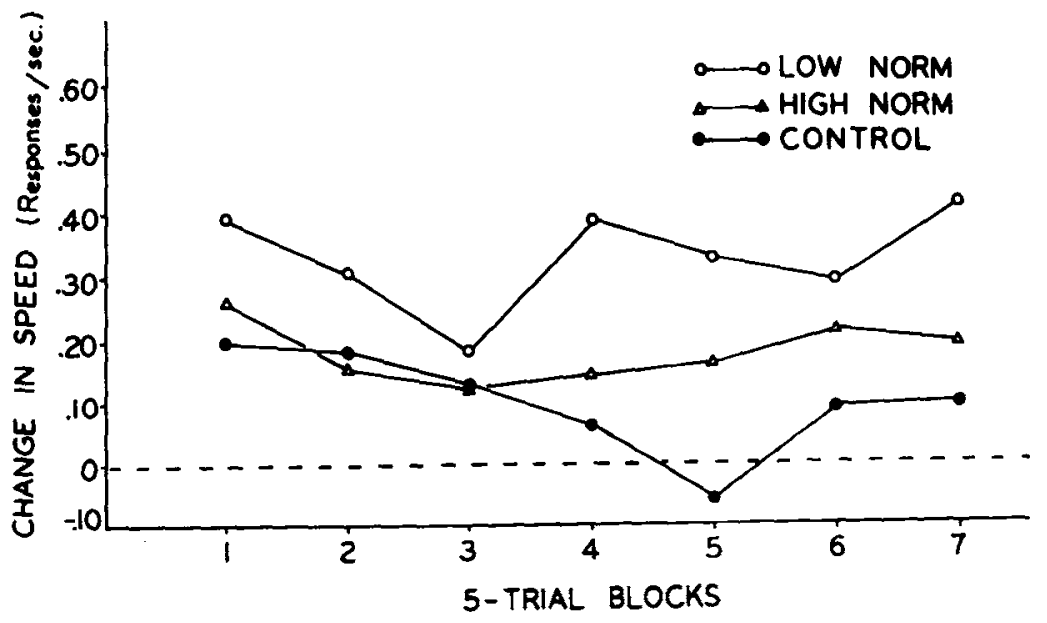


Fig. 4. Mean change in lever-pressing speed for the fast responders in each group as a function of seven five-trial blocks.

block. This increment decays rapidly for the control group Ss so that by Trial Block 4, SLO responders in the control condition are, on the average, approximately at their initial baseline speed. Both norm conditions show speed scores which increase over the course of the 35-trial session.

FST responders, on the other hand (Fig. 4), appear to be relatively insensitive to the treatment conditions. The condition having the smallest overall mean increment in speed over the entire course of the 35-trial session is the high-norm condition.

The data for the ITM group (Fig. 3) suggest a pattern halfway between that of the FST and SLO Ss in that the initial increment in the response speed decays in the control condition. The effect of the high-norm condition appears relatively stable over the 35 trials, while the low-norm condition gives rise to the largest increment in response speed.

The data indicate that the low-norm condition resulted in greater increase in speed than did the high-norm condition for Ss in the FST and ITM groups. This pattern is reversed for the last four trial blocks of the SLO group.

The data shown in Figs. 2, 3, and 4 were submitted to separate analyses of variance. The effect of the treatment condition is limited to the slowest third of the experimental sample, as no main or interaction effects were reliable in either the FST or the ITM conditions $(p<.25)$. The analysis of variance for the SLO group indicates a significant main effect of norm

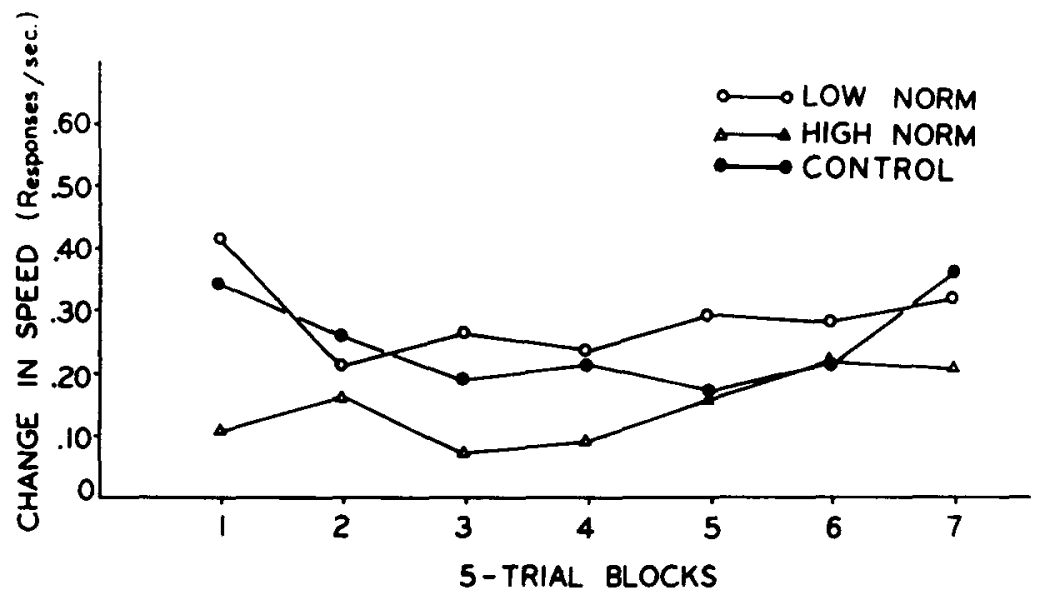

$[F(2,27)=4.04, p<.05]$ and a significant Trials by Norm interaction $[F(12,162)=2.56, p<.01]$. Application of the Duncan multiple-range test indicates that the means associated with the low-norm condition significantly exceed those associated with the control condition after Trial Block $2(p<.05)$, while the means associated with the high-norm condition significantly exceed those associated with the control condition after Trial Block $3(p<.05)$.

The results indicate that an externally administered norm appears to have an enhancing effect upon the performance of slow responders when compared with a control condition, whereas easy or unattainable norms, within the limits used in the study, do not appear to result in a performance level that is significantly greater than control for fast responders. The results also indicate that there is a tendency for an attainable norm to give rise to greater increment in speed than an unattainable norm, although the difference was not reliable.

\section{REFERENCES}

LEUBA, C. Towards some integration of learning theories: The concept of optimal stimulation. Psychological Reports, 1955, 22, 27-33.

SWINGLE, P. G., COADY, H., \& MOORS, D The effects of performance feedback, social and monetary incentive upon human lever pressing rate. Psychonomic Science, 1966, 4, 209-210.

SWINGLE, P. G., \& MOORS, D. The effects of small monetary incentive upon human lever pressing rates. Psychonomic Science, 1967, 9, 205-206.

\section{NOTES}

1. This research was supported by a grant from the Department of National Health and Welfare of Canada.

2. As the high-norm condition was designed to be impossible to achieve, any $S$ exceeding the norm during the 35 trials was dropped and replaced. One $S$ who would have been classified as a slow responder did pass the high norm. He was dropped from the study and replaced by another $S$. 